\title{
Caractérisation des souches de Pseudocercosporella herpotrichoides, agent du piétin-verse des céréales, résistantes au prochloraze, isolées en France sur blé tendre d'hiver
}

\author{
P Leroux 1, P Marchegay 2,* \\ 1 INRA, station de phytopharmacie, route de Saint-Cyr, 78026 Versailles Cedex; \\ ${ }^{2}$ ACTA, 149 rue de Bercy, 75595 Paris cedex 12, France
}

(Reçu le 23 mai 1991; accepté le 27 août 1991)

\begin{abstract}
Résumé - Des souches de $P$ herpotrichoides résistantes au prochloraze ont été isolées pour la première fois en France au cours de l'année 1990 sur des tiges de blé prélevées dans 3 parcelles ayant reçu au moins un traitement à base de prochloraze. Les niveaux de résistance de ces nouveaux phénotypes qui sont tous caractérisés par une croissance mycélienne lente sur milieu nutritif gélosé, sont de l'ordre de 20-30. Ces nouvelles souches peuvent présenter une sensibilité réduite (ex: imazalil, flusilazole, flutriafol, propiconazole, tébuconazole) ou accrue (ex : triflumizole, fénarimol, cyproconazole, hexaconazole, triadiménol) vis-à-vis d'autres inhibiteurs de la C-14 déméthylation des stérols, ceci comparativement aux souches à croissance lente isolées antérieurement. À la différence de mutants induits en laboratoire et résistants au prochloraze, ces souches naturelles ne présentent pas une sensibilité réduite visà-vis de la terbinafine, un inhibiteur de la squalène-époxydase. Les conséquences du développement au champ de tels phénotypes de $P$ herpotrichoides résistants au prochloraze sont discutées.
\end{abstract}

inhibiteur de la biosynthèse des stérols / résistance croisée / niveaux de résistance / flusilazole

Summary - Resistance to prochloraz in strains of the cereal eyespot fungus Pseudocercosporella herpotrichoides isolated from winter wheat in France. Several prochloraz-resistant strains of $\mathrm{P}$ herpotrichoides were isolated for the first time in France during the year 1990 on winter wheat stems taken from 3 separate plots which had been previously treated with prochloraz. At sites 1 and 2 in the North of France the efficiency of prochloraz was found to be reduced. At site 3 where a long-term trial was taking place, prochloraz was found to be highly effective (table 11$)$. The resistance levels of these new phenotypes (IIp-type), which all produced slow-growing colonies (II-or $R$-type), were $\approx$ 20-30 in tests on mycelial growth or germ tube elongation (tables I and II; figs 1 and 2). Compared to the slowgrowing strains isolated previously (Ils-type), the new strains were more tolerant towards imazalil, flusilazol, flutriafol, propiconazole and tebuconazole, more sensitive towards triflumizol, fenarimol, cyproconazole, hexaconazole and triadimenol, and equally sensitive towards clotrimazole, dichlobutrazol and diniconazole (table II). These fungicides, like prochloraz, are inhibitors of sterol C-14 demethylation. The prochloraz-resistant strains did not exhibit crossresistance towards terbinafine and fenpropimorph which respectively inhibit the squalene-epoxidase and the sterol $\Delta 14$ reductase or $\Delta 8 \rightarrow \Delta 7$ isomerase (tables $I V$ and $V$ ). The practical consequences of the development of such prochloraz-resistant strains of $\mathrm{P}$ herpotrichoides in winter wheat are discussed.

sterol biosynthesis inhibitor / cross-resistance / resistance level / flusilazol

\footnotetext{
- Adresse actuelle : INRA, station de pathologie végétale, route de Saint-Cyr, 78026 Versailles Cedex, France
} 


\section{INTRODUCTION}

Le piétin-verse des céréales provoqué par Pseudocercosporella herpotrichoides est une maladie importante notamment sur blé d'hiver. La lutte chimique contre ce parasite est possible grâce à 1 ou 2 applications de fongicides systémiques ou pénétrants, réalisées courant tallagemontaison. Pendant une dizaine d'années, les matières actives les plus utilisées ont été le carbendazime ou ses précurseurs : le bénomyl et le thiophanate-méthyle. Ces fongicides qui appartiennent à la famille des benzimidazoles empêchent la formation des microtubules du fuseau achromatique et de ce fait, bloquent les mitoses chez les champignons (Leroux, 1987). À partir de 1981, en France et dans divers autres pays européens, des souches de $P$ herpotrichoides fortement résistantes à ces benzimidazoles ont été détectées et ont rapidement supplanté les souches sensibles dans les zones de culture intensive du blé. Dans les parcelles où ces souches résistantes deviennent prédominantes, l'efficacité pratique des traitements à base de benzimidazoles peut être nulle (Leroux et Cavelier, 1983; King and Griffin, 1985; Fitt et al, 1990).

Face à cette situation, la seule solution a été le remplacement des benzimidazoles par des fongicides inhibiteurs de la biosynthèse des stérols. La première matière active autorisée a été un imidazole : le prochloraze et par la suite un triazole a été introduit : le flusilazole. Ces 2 fongicides inhibent la même cible au niveau de la biosynthèse des stérols : la $\mathrm{C}-14$ déméthylase. Dans une précédente étude, nous avons montré que des souches de $P$ herpotrichoides collectées en France entre 1984 et 1987 présentaient une variabilité de sensibilité in vitro au flusilazole et plus généralement à tous les fongicides inhibiteurs de la biosynthèse des stérols, comportant l'hétérocycle "triazole" (Leroux et Gredt, 1988). Nous avons alors distingué 3 phénotypes (tableau 1):

- la : souches à croissance rapide, sensibles aux "triazoles";

- lb : souches à croissance rapide, résistantes aux "triazoles";

- II : souches à croissance lente, résistantes aux «triazoles».

L'ensemble de ces phénotypes est sensible au prochloraze. Dans la pratique, cette variabilité fait que, pour les sites où les phénotypes la sont dominants, les efficacités du fluzilazole et du prochloraze sont équivalentes, alors que là où les phénotypes II sont majoritaires, seul le prochloraze présente une activité correcte (Curé et al, 1988; Migeon et Copin, 1988).

Ces observations ont conduit bon nombre de céréaliculteurs à choisir le prochloraze comme produit de base contre le piétin-verse. Bien évidemment l'usage intensif d'un tel fongicide à site d'action unique constitue une condition favorable au développement de souches résistantes. Pour

Tableau I. Caractéristiques et nomenclature des diverses souches de Pseudocercosporella herpotrichoides.

$\begin{array}{lll}\begin{array}{l}\text { Croissance } \\ \text { mycélienne }\end{array} & \text { Variété (b) } & \begin{array}{c}\text { Pouvoir pathogène } \\ \text { important sur à des inhibiteurs de la biosynthèse des stérols }\end{array}\end{array}$ Nomenclature (d)

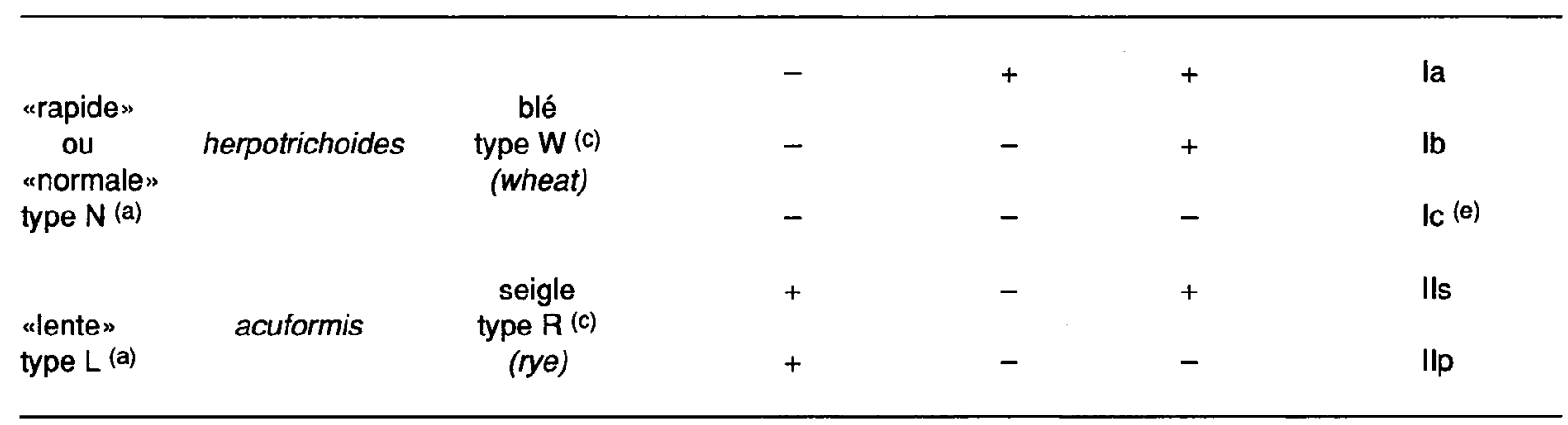

(a) nomenclature de Cavelier et al (1987); (b) dénomination retenue par Nirenberg (1981); (c) appellation proposée par Lange de la Camp (1966), puis reprise par Scott et al (1975); (d) nomenclature basée sur celle de Leroux et Gredt (1988) et modifiée du fait de la détection au champ de souches résistantes au prochloraze (type Ilp); (e) mutant induits en laboratoire. 
tenter de prévoir ce risque, une démarche consiste à induire au laboratoire des mutants résistants au prochloraze. De tels mutants, qui d'ailleurs résistent simultanément à ce fongicide et aux triazoles ont effectivement été obtenus à partir de souches de type la (Leroux et al, 1988). Grâce à ces mutants (type lc; tableau I) nous avons pu élaborer une méthode permettant d'éventuellement les détecter dans des populations naturelles. Ainsi, dans des essais conduits sur spores à des concentrations de prochloraze comprises entre 0,01 et $0,05 \mathrm{mg} / \mathrm{l}$, les filaments germinatifs apparaissent longs pour les mutants Ic et très courts pour les souches la, lb et II. Or, pour la première fois en 1990 nous avons décelé des isolats naturels ayant une réaction proche de celle des mutants de type Ic, en présence de prochloraze. Dans cette publication, nous présentons les caractéristiques de ce nouveau phénotype de $P$ herpotrichoides résistant au prochloraze.

\section{MATÉRIELS ET MÉTHODES}

Les souches résistantes au prochloraze proviennent de 3 parcelles de blé tendre d'hiver ayant reçu en 1990, 1 ou 2 traitements à base de prochloraze (tableau II). Les isolements ont été réalisés après l'épiaison, au moment de la formation des grains, à partir de symptômes caractéristiques de piétin-verse sur tige, selon la technique de Leroux et Gredt (1988). Toutes les souches récoltées présentent une croissance lente sur milieu nutritif gélosé et sont résistantes aux benzimidazoles. Au vu de ces caractéristiques, nous avons choisi de regrouper ces souches sous le vocable "type Ilp". Quant aux autres souches à croissance lente sensibles au prochloraze, nous les avons regroupées au sein du type lis qui équivaut au type II défini précédemment (tableau I; Leroux et Gredt, 1988; Leroux et al, 1988). Le comportement des souches de type IIp est comparé à celui des souches de type la, lb et lis collectées dans la nature sur blé d"hiver, entre 1984 et 1987. Par ailleurs, 2 mutants de laboratoire (M.tl et AS.tl du type Ic) présentant une croissance rapide et résistants à divers inhibiteurs de la biosynthèse des stérols dont le prochloraze (Leroux et al, 1988) sont inclus dans la présente étude. Toutes les souches étudiées sont conservées sur du malt-agar (cristomalt $10 \mathrm{~g}$; agar-agar $12,5 \mathrm{~g}$ pour 1 I) dépourvu de tout fongicide.

Les divers types d'inhibiteurs de la biosynthèse des stérols expérimentés (Leroux et Gredt, 1988), sont sous forme de produits techniques titrant au minimum $90 \%$ de matière active. Lors des essais fongicides, ils sont incorporés dans les milieux de culture, sous forme de solutions éthanoliques, la concentration finale d'alcool dans le milieu étant de 0,25\%.

L'effet des fongicides sur la croissance mycélienne de $P$ herpotrichoides est étudié selon la méthode de
Leroux et Gredt (1988). Le milieu de culture, dont la composition est la suivante pour 1 I d'eau désionisée : $2 \mathrm{~g}$ de $\mathrm{KH}_{2} \mathrm{PO}_{4} ; 1,5 \mathrm{~g}$ de $\mathrm{K}_{2} \mathrm{HPO}_{4} ; 1 \mathrm{~g}$ de $\left(\mathrm{NH}_{4}\right)_{2} \mathrm{SO}_{4}$; $0,5 \mathrm{~g}$ de $\mathrm{Mg} \mathrm{SO}_{4}, 7 \mathrm{H}_{2} \mathrm{O} ; 2 \mathrm{~g}$ d'extrait de levure (Difco); $10 \mathrm{~g}$ de glucose et $12,5 \mathrm{~g}$ d'agar-agar, a été préféré au PDA (potato dextrose agar) ou au maltagar car sur ces 2 derniers milieux les souches à croissance lente ont un développement particulièrement faible. Les essais sont réalisés à $19^{\circ} \mathrm{C}$ et à l'obscurité. Le diamètre des plages mycéliennes est mesuré tous les 7 j pendant 3 semaines. Pour chaque fongicide, on établit une courbe de réponse à partir d'au moins 5 concentrations, ce qui conduit à la détermination de la concentration inhibant la vitesse de croissance mycélienne de $50 \%\left(\mathrm{Cl}_{50}\right.$ «mycélium») et de la concentration minimale inhibitrice (CMI).

Pour étudier l'action des fongicides sur l'élongation des filaments germinatifs émis par les spores de $P$ herpotricoides, le milieu employé est l'eau gélosée (12,5 g agar-agar/l). Le protocole expérimental choisi correspond à celui décrit précédemment par Leroux et Gredt (1988). Après une incubation à $19^{\circ} \mathrm{C}$ pendant $48 \mathrm{~h}$, la mesure de la longueur des filaments germinatifs apicaux permet d'estimer les concentrations inhibant leur élongation de $50 \%\left(\mathrm{Cl}_{50}\right.$ "filament") ou de $90 \%\left(\mathrm{Cl}_{90}\right.$ «filament»).

Des niveaux de résistance peuvent être calculés en faisant le rapport :

$\mathrm{Cl}_{50}$ "mycélium» ou «filament» souches résistantes

$\mathrm{Cl}_{50}$ «mycélium» ou «filament» souches sensibles

\section{RÉSULTATS}

Les essais conduits sur la croissance mycélienne des souches de $P$ herpotrichoides collectées entre 1984 et 1987 indiquent que, pour le prochloraze les $\mathrm{Cl}_{50}$ «mycélium» sont comprises entre 0,03 et $0,07 \mathrm{mg} / \mathrm{l}$ et que les $C M /$ sont toujours inférieures à $1 \mathrm{mg} / \mathrm{l}$. Chez les souches isolées en 1990, l'inhibition est observée à des concentrations nettement supérieures aux précédentes, puisque les $\mathrm{Cl}_{50}$ "mycélium» se situent entre 0,6 et $0,9 \mathrm{mg} / \mathrm{l}$ et les $C M /$ sont supérieures à $5 \mathrm{mg} / \mathrm{l}$. Quant aux 2 mutants induits en laboratoire, leur comportement est intermédiaire, avec des valeurs respectives des $\mathrm{Cl}_{50}$ «mycélium» et des $C M I$ voisines de 0,25 et 2,5 $\mathrm{mg} / \mathrm{l}$ (tableau III, fig 1).

Lors des essais de germination des spores, les souches naturelles de $P$ herpotrichoides présentent 2 types de comportement en présence de prochloraze. Les plus sensibles dont les $\mathrm{Cl}_{50}$ «filament» sont comprises entre 0,002 et 0,006 $\mathrm{mg} / \mathrm{l}$ et correspondent aux souches collectées entre 1984 et 1987. Quant à celles isolées en 


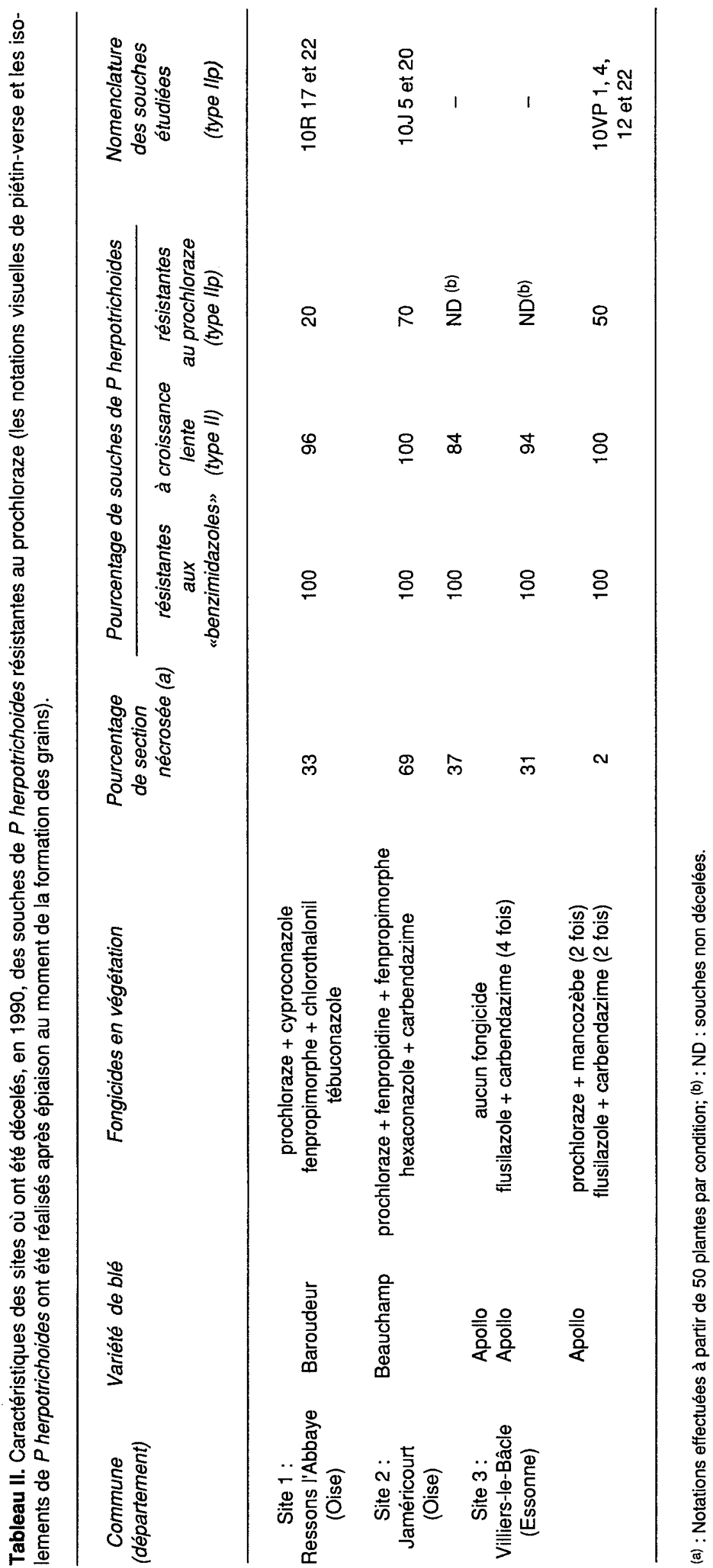


Tableau III. Effet du prochloraze sur la croissance mycélienne et l'élongation des filaments germinatifs de diverses souches de $P$ herpotrichoides (concentrations en $\mathrm{mg} / \mathrm{l}$ estimées à partir de 3 expériences indépendantes).

\begin{tabular}{|c|c|c|c|c|c|}
\hline \multirow{2}{*}{ Souche (a) } & \multirow{2}{*}{ Phénotype } & \multicolumn{2}{|c|}{ Croissance mycélienne } & \multicolumn{2}{|c|}{ Filaments germinatifs } \\
\hline & & $\mathrm{Cl}_{50}^{(b)}$ & $C M I(c)$ & $\mathrm{Cl}_{50}{ }^{(b)}$ & $\mathrm{Cl}_{90}{ }^{(d)}$ \\
\hline 10R.17 & $\| p$ & 0,8 & 10 & 0,06 & 0,5 \\
\hline 10R.22 & IIp & 0,8 & 8 & 0,06 & 0,6 \\
\hline 10J.5 & $\| l p$ & 0,7 & 10 & 0,05 & 0,4 \\
\hline 10J.20 & $\| p$ & 0,9 & 12 & 0,06 & 0,5 \\
\hline 10VP.1 & $\| p$ & 0,7 & 10 & 0,05 & 0,4 \\
\hline 10VP.4 & $\| p$ & 0,8 & 12 & 0,06 & 0,6 \\
\hline 10VP.12 & lip & 0,7 & 10 & 0,07 & 0,4 \\
\hline 10VP.22 & $\| i p$ & 0,6 & 8 & 0,08 & 0,6 \\
\hline $4 S .6$ & Ils & 0,03 & 0,6 & 0,003 & 0,02 \\
\hline $5 \mathrm{~B} .21$ & Ils & 0,04 & 0,7 & 0,002 & 0,03 \\
\hline $5 Q .22$ & Ils & 0,03 & 0,6 & 0,002 & 0,02 \\
\hline 7AR.22 & Ils & 0,06 & 0,6 & 0,003 & 0,02 \\
\hline 4AS.1 & la & 0,04 & 0,5 & 0,003 & 0,02 \\
\hline 5L.10 & la & 0,05 & 0,6 & 0,004 & 0,03 \\
\hline $8 P .6$ & la & 0,07 & 0,7 & 0,005 & 0,04 \\
\hline $5 B .1$ & lb & 0,07 & 0,6 & 0,004 & 0,03 \\
\hline $5 A Q .25$ & lb & 0,07 & 0,5 & 0,003 & 0,03 \\
\hline $7 G .13$ & $\mathrm{lb}$ & 0,06 & 0,6 & 0,006 & 0,05 \\
\hline M.t1 & Ic & 0,2 & 2,0 & 0,2 & 0,7 \\
\hline AS.t1 & ic & 0,3 & 3,0 & 0,3 & 0,8 \\
\hline
\end{tabular}

(a) Les souches 5B.1, 5AQ.25 et 7G.13 appartenant au phénotype lb ont été choisies parmi les plus résistantes aux triazoles (Leroux et Gredt, 1988); (b) $\mathrm{Cl}_{50}$ : concentrations inhibant de $50 \%$ la vitesse de croissance mycélienne ou l'élongation des filaments germinatifs apicaux. ${ }^{(c)} \mathrm{CMI}$ : concentrations minimales inhibitrices de la croissance mycélienne (au bout de 3 semaines d'incubation); (d) $\mathrm{Cl}_{90}$ : concentrations inhibant de $90 \%$ l'élongation des filaments germinatifs apicaux.

1990, elles sont moins affectées par le prochloraze puisque leurs $\mathrm{Cl}_{50}$ «filament" varient entre 0,05 et $0,08 \mathrm{mg} / \mathrm{l}$. II convient de noter que dans ce type d'essai les mutants de laboratoire apparaissent plus résistants au prochloraze que les souches naturelles $\left(\mathrm{Cl}_{50}\right.$ "filament" voisines de $0,25 \mathrm{mg} / \mathrm{l}$ ) (tableau III, fig 2).

Parallèlement aux investigations conduites avec le prochloraze, nous avons analysé l'effet de 3 autres inhibiteurs de la $\mathrm{C}-14$ déméthylation des stérols renfermant aussi un hétérocycle «imidazole». L'imazalil présente un comportement voisin de celui du prochloraze, notamment dans les essais sur la germination des spores. En effet, les souches de type IIp apparaissent plus tolérantes à l'imazalil que celles de type lls. Cette différence ne se retrouve pas avec le clotrimazole qui affecte de la même manière toutes les souches à croissance lente. Quant au triflumizole, il présente la particularité d'être beaucoup plus toxique pour les souches de type Ilp que pour celles de type Ils; les $\mathrm{Cl}_{50}$ efilament» sont respectivement de l'ordre de 0,1 et $2 \mathrm{mg} / \mathrm{l}$. Cette sensibilité accrue des phénotypes IIp vis-à-vis du triflumizole est également observée dans les

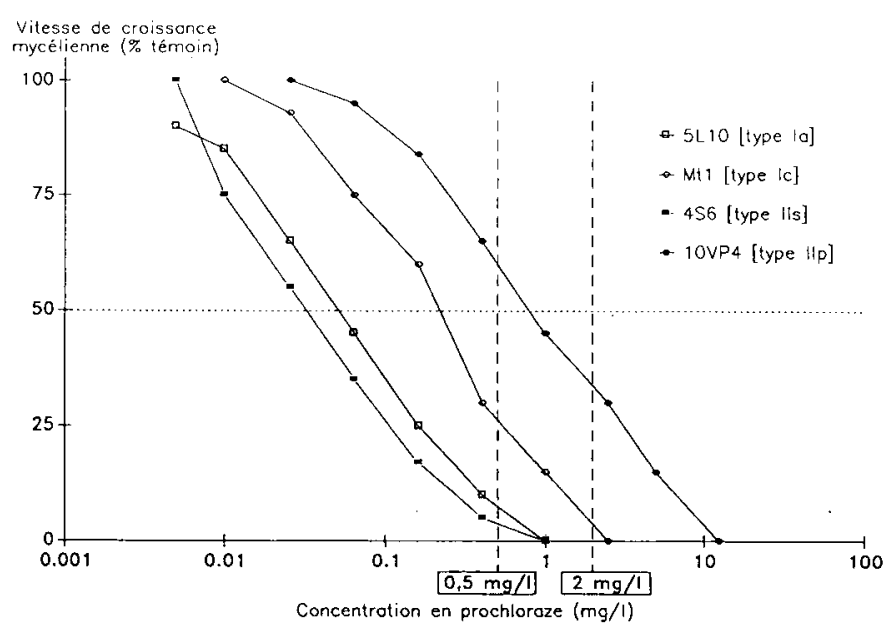

Fig 1. Effet du prochloraze sur la vitesse de croissance mycélienne de différentes souches de $P$ herpotrichoides. 


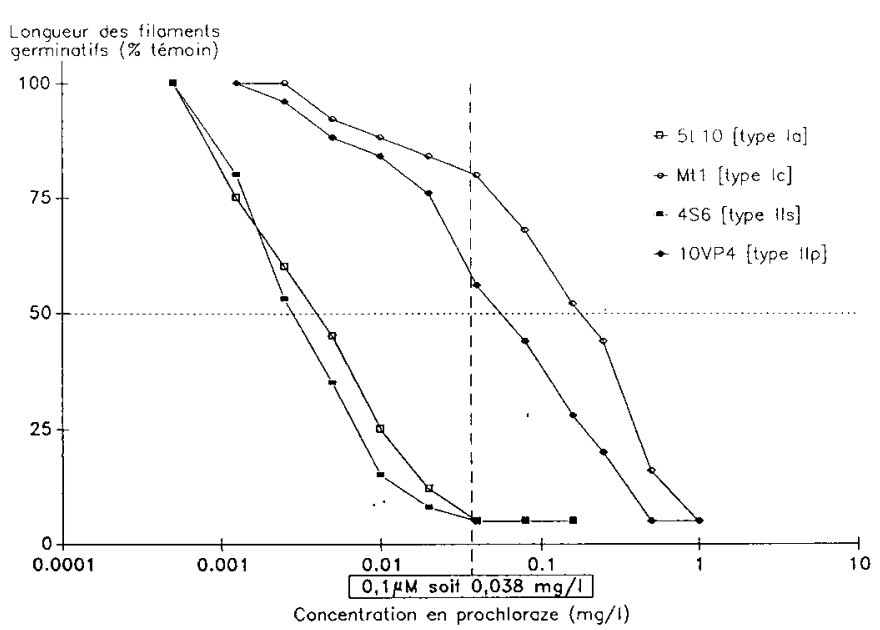

Fig 2. Effet du prochloraze sur l'élongation des filaments germinatifs apicaux émis par les spores de différentes souches de $P$ herpotrichoides.

essais sur mycélium. Le comportement de ces 3 «imidazoles» est similaire chez les souches à croissance rapide. En effet celles de types lb et lc apparaissent plus tolérantes à ces fongicides que celles de type la (tableaux IV et V).
Les inhibiteurs de la $\mathrm{C}-14$ déméthylation des stérols comportant un hétérocycle triazole peuvent être répartis en 3 catégories selon leurs effets au niveau de l'élongation des tubes germinatifs des souches à croissance lente. Le flusilazole, le flutriafol, le propiconazole et le tébuconazole sont moins toxiques pour les souches de type llp que pour celles de type lis. À l'opposé, en présence de cyproconazole, d'hexaconazole ou de triadiménol, ce sont les souches de type Ilp qui apparaissent les plus sensibles. Quant au diniconazole et au diclobutrazol, ils présentent une activité similaire chez toutes les souches à croissance lente (tableau IV). Dans les essais conduits sur mycélium avec le flusilazole, la distinction entre les types Ils et IIp est difficile à faire sur la base des $\mathrm{Cl}_{50}$ mycélium; par contre, avec les CMI, il se confirme que les souches de type IIp sont plus tolérantes à ce fongicide que les souches de type IIs (tableau V).

Deux autres types d'inhibiteurs de la biosynthèse des stérols ont également été expérimentés; ce sont d'une part, le fenpropimorphe qui bloque la $\Delta 14$ réductase et/ou la $\Delta 8 \rightarrow \Delta 7$ isomérase, et d'autre part, la terbinafine qui bloque

Tableau IV. Effet de différents inhibiteurs de la biosynthèse des stérols sur l'élongation des filaments germinatifs de diverses souches de $P$ herpotrichoides (concentrations en $\mathrm{mg} / \mathrm{l}$ estimées à partir de 2 ou 3 expériences indépendantes).

\begin{tabular}{|c|c|c|c|c|c|c|c|c|c|c|}
\hline \multirow{3}{*}{ Fongicide } & \multicolumn{10}{|c|}{ Phénotypes de $\mathrm{P}$ herpotrichoides } \\
\hline & \multicolumn{2}{|c|}{$\begin{array}{l}l a \\
(3)(a)\end{array}$} & \multicolumn{2}{|r|}{$\begin{array}{l}l b \\
(3)\end{array}$} & \multicolumn{2}{|c|}{$\begin{array}{c}l c \\
(2)\end{array}$} & \multicolumn{2}{|c|}{$\begin{array}{l}\text { IIs } \\
\text { (3) }\end{array}$} & \multicolumn{2}{|c|}{$\begin{array}{l}I / p \\
(6)\end{array}$} \\
\hline & $\mathrm{Cl}_{50}{ }^{\text {(b) }}$ & $C I_{50}{ }^{(b)}$ & $\mathrm{Cl}_{50}$ & $\mathrm{Cl}_{90}$ & $\mathrm{Cl}_{50}$ & $\mathrm{Cl}_{90}$ & $\mathrm{Cl}_{50}$ & $\mathrm{Cl}_{90}$ & $\mathrm{Cl}_{50}$ & $\mathrm{Cl}_{90}$ \\
\hline Prochloraze & 0,004 & 0,03 & 0,004 & 0,04 & 0,25 & 0,75 & 0,002 & 0,02 & 0,06 & 0,5 \\
\hline Clotrimazole & 0,15 & 1,0 & 0,4 & 2,5 & 4,0 & $>10$ & 0,2 & 1,0 & 0,25 & 1,2 \\
\hline Imazalil & 0,002 & 0,05 & 0,03 & 0,3 & 0,4 & 1,5 & 0,02 & 0,20 & 0,15 & 1,0 \\
\hline Triflumizole & 0,05 & 0,4 & 3,0 & 25 & 3,0 & 15 & 2,0 & 10 & 0,10 & 1,0 \\
\hline Fénarimol & 0,1 & 1,5 & 2,5 & $>10$ & 4 & $>10$ & 1,0 & 2,5 & 0,35 & 1,5 \\
\hline Cyproconazole & 0,4 & 3,0 & 2,0 & $>10$ & 9 & $>10$ & 0,6 & 4 & 0,6 & 4 \\
\hline Diclobutrazol & 0,4 & 3,0 & 2,0 & $>10$ & 9 & $>10$ & 0,6 & 4 & 0,6 & 4 \\
\hline Diniconazole & 0,1 & 0,9 & 0,5 & 2,5 & 4 & 9 & 0,2 & 2 & 0,2 & 1,5 \\
\hline Flusilazole & 0,005 & 0,10 & 0,08 & 0,5 & 1,7 & $>5,0$ & 0,06 & 0,5 & 0,3 & 1,2 \\
\hline Flutriafol & 0,15 & 0,8 & 1,0 & 6,0 & 16 & $>25$ & 0,6 & 4 & 2,5 & 20 \\
\hline Hexaconazole & 0,01 & 0,2 & 0,4 & 2,5 & 0,9 & 4 & 0,3 & 0,8 & 0,1 & 0,5 \\
\hline Propiconazole & 0,02 & 0,3 & 0,6 & 1,5 & 1,2 & 7 & 0,25 & 1 & 0,8 & 5 \\
\hline Tébuconazole & 0,03 & 0,2 & 1,5 & 8 & 1,0 & 3,5 & 0,5 & 1,5 & 1,3 & 4,5 \\
\hline Triadiménol & 0,3 & 3,5 & 40 & $>50$ & 18 & $>50$ & 15 & $>50$ & 5 & 40 \\
\hline Terbinafine & 0,008 & 0,10 & 0,012 & 0,10 & 0,4 & $>1$ & 0,012 & 0,13 & 0,015 & 0,15 \\
\hline
\end{tabular}

(a) Nombre de souches étudiées par phénotype; (b) $\mathrm{Cl}_{50}$ et $\mathrm{Cl}_{90}$ : concentrations inhibant respectivement de $50 \%$ et $90 \%$ l'élongation des filaments germinatifs apicaux. 
Tableau V. Effet de différents inhibiteurs de la biosynthèse des stérols sur la croissance mycélienne de diverses souches de $P$ herpotrichoides (concentrations en $\mathrm{mg} / \mathrm{l}$ estimées à partir de 2 ou 3 expériences indépendantes).

Phénotypes de $\mathrm{P}$ herpotrichoides

\begin{tabular}{|c|c|c|c|c|c|c|c|c|c|c|}
\hline \multirow[t]{2}{*}{ Fongicide } & \multicolumn{2}{|c|}{${ }_{(3)^{(a)}}$} & \multicolumn{2}{|r|}{$\begin{array}{l}16 \\
(3)\end{array}$} & \multicolumn{2}{|c|}{$\begin{array}{r}l c \\
(2)\end{array}$} & \multicolumn{2}{|c|}{$\begin{array}{l}\text { IIs } \\
\text { (3) }\end{array}$} & \multicolumn{2}{|c|}{$\begin{array}{l}\| p \\
(6)\end{array}$} \\
\hline & $C I_{50}^{(b)}$ & $C M I(c)$ & $\mathrm{Cl}_{50}$ & $C M I$ & $\mathrm{Cl}_{50}$ & $C M I$ & $\mathrm{Cl}_{50}$ & $C M I$ & $\mathrm{Cl}_{50}$ & $C M I$ \\
\hline Prochloraze & 0,05 & 0,6 & 0,07 & 0,6 & 0,25 & 2,5 & 0,03 & 0,6 & 0,80 & 10 \\
\hline Clotrimazole & 0,6 & 10 & 1,2 & 12 & 3,2 & 30 & 1,0 & 10 & 0,8 & 9,0 \\
\hline Imazalil & 0,03 & 0,4 & 0,3 & 2 & 0,4 & 2,5 & 0,3 & 3 & 1,2 & 15 \\
\hline Triflumizole & 0,4 & 8 & 20 & $>50$ & 2,5 & 23 & 35 & $>50$ & 1,2 & $>50$ \\
\hline Flusilazole & 0,06 & 0,8 & 0,4 & 3,2 & 0,8 & $>5$ & 0,4 & 3,5 & 0,8 & $>5$ \\
\hline Terbinafine & 0,15 & 5,0 & 0,10 & 4,0 & 0,9 & 13 & 0,15 & 3,0 & 0,17 & 3,0 \\
\hline Fenpropimorphe & 13 & $>25$ & 15 & $>25$ & 15 & $>25$ & 1,8 & $>25$ & 2,2 & $>25$ \\
\hline
\end{tabular}

(a) Nombre de souches étudiées par phénotype; (b) $\mathrm{Cl}_{50}$ : concentration inhibant de $50 \%$ la vitesse de croissance mycélienne; (c) $C M l$ : concentration minimale inhibitrice de la croissance mycélienne (au bout de 3 semaines d'incubation).

la squalène-époxydase (Leroux et Benveniste, 1985). Tout d'abord, vis-à-vis du fenpropimorphe, toutes les souches à croissance lente (types Ils et IIp) apparaissent nettement plus sensibles que l'ensemble des souches à croissance rapide (types la, lb et Ic). Avec la terbinafine les essais conduits tant sur mycélium que sur spores indiquent que toutes les souches naturelles présentent une sensibilité équivalente. Par contre, nous confirmons que les mutants de type ic sont tolérants vis-à-vis de cet inhibiteur de la squalèneépoxydase (tableaux IV et V).

\section{CONCLUSIONS ET DISCUSSION}

Il a donc fallu comme dans le cas des fongicides benzimidazoles, une dizaine d'années d'utilisation du prochloraze pour détecter des souches de $P$ herpotrichoides résistantes à cet inhibiteur de la biosynthèse des stérols. Pour les benzimidazoles, nous avons répertorié 6 phénotypes résistants, mais en fait 2 sont prédominants et présentent des niveaux de résistance très élevés (supérieurs à 1000 vis-à-vis du carbendazime) (Leroux et Gredt, 1988). Cette résistance aux benzimidazoles concerne aussi bien les souches à croissance rapide (type I) que celles à croissance lente (type II). Pour l'instant, dans le cas du prochloraze, la résistance n'a été décelée dans la nature que chez des souche sance lente (type IIp). Toutefois, le développement de souches à croissance rapide, résistantes au prochloraze n'est pas à exclure; d'autant qu'en laboratoire de tels phénotypes ont pu être induits (type $\mathrm{Ic}$ ). Les niveaux de résistance des souches de type IIp, vis-à-vis du prochloraze sont de l'ordre de 20 à 30 (estimations à partir des $\mathrm{Cl}_{50}$ "mycélium" ou "filament", comparativement à des souches de type IIs). A la différence de phénotypes lb qui, vis-à-vis des triazoles présentent une variabilité de comportement (Leroux et Gredt, 1988), il s'avère que, quelle que soit leur origine, les souches de type Ilp ont des réactions similaires vis-à-vis du prochloraze. En pratique, ceci pourrait conduire à des évolutions différentes des populations de $P$ herpotrichoides selon la nature de l'inhibiteur de la C-14 déméthylation des stérols qui leur serait appliqué. Ainsi, dans une population ne comportant que des souches à croissance rapide (type I), l'évolution pourrait être de type progressif (ou directionnel) en présence de triazoles, du fait de la multiplicité des phénotypes résistants lb. Un tel comportement est fréquent avec les inhibiteurs de la C-14 déméthylation des stérols (Koller et Scheinpflug, 1987; Koller, 1988; Leroux, 1987 et 1989); l'exemple le plus connu concerne l'oïdium de l'orge (Butters et al, 1984; Andrivon et al, 1987; Heanay, 1988). Par contre, si une population ne renfermant que des souches à croissance lente (type II) est traîtée avec du prochloraze, une évolution de type disruptif est pré- 
visible (Koller et Scheinpflug, 1987). De tels cas de résistance concernent les benzimidazoles, notamment vis-à-vis de $P$ herpotrichoides (Leroux, 1987 et 1989). Par ailleurs, comme toute possibilité de transport des souches de type Ilp, entre les parcelles prospectées en 1990, semble exclue, il est probable qu'elles se soient développées simultanément en plusieurs sites. Une hypothèse similaire a été envisagée pour expliquer le développement des souches de $P$ herpotrichoides résistantes aux benzimidazoles dans de nombreuses zones céréalières européennes au début des années 1980.

Étant donné que nous n'avons pas isolé de souches de type llp à partir des nombreux échantillons analysés dans notre laboratoire avant 1990 , nous pouvons penser que leur sélection est un processus relativement récent. Cette hypothèse est renforcée par le fait que dans un essai à long terme (Site 3; Marchegay et al, 1988), aucune souche de type llp n'avait été décelée entre 1987 et 1989, (Leroux et Marchegay, résultats non publiés). Quant aux observations réalisées sur ce site en 1990, elles indiquent, d'une part, que le flusilazole sélectionne moins fortement les souches de type llp que le prochloraze et, d'autre part, que la résistance en pratique à ce dernier fongicide n'existe pas (tableau II). En effet, une résistance en pratique ne peut être diagnostiquée que si 2 conditions sont remplies simultanément : présence de souches résistantes et réduction d'efficacité (Leroux, 1987). Or, en 1990, l'activité de prochloraze est tout-à-fait correcte. En ce qui concerne les 2 autres sites où des souches de type Ilp ont été décelées, les informations dont nous disposons sont insuffisantes pour conclure à une éventuelle résistance en pratique du prochloraze.

Il est pourtant tentant de l'affirmer dans le site 2 (tableau II), où une forte fréquence des souches de type Ilp est associée à une infestation importante de piétin-verse. En fait, il faut être prudent car des prospections dans la même région nous ont montré que des activités médiocres du prochloraze peuvent être constatées sans qu'il y ait de souches de type lip (Leroux et Paulmier, résultats non publiés). Toutefois, certaines caractéristiques des souches de type $\mathrm{llp}$ : $\mathrm{Cl}_{50}$ «mycélium» comprises entre 0,6 et $0,9 \mathrm{mg} / \mathrm{l}$ de prochloraze et niveaux de résistance supérieurs à 10 (comparativement aux souches de type lls et la), laissent présager une résistance en pratique à ce fongicide en cas de généralisation des phénotypes Ilp. Notre crainte est basée sur le fait qu'avec le flusilazole, l'efficacité pratique est médiocre dans les situations où les souches de type lls dominent (ex : site 3 , tableau 1) alors qu'elle est correcte et équivaut à celle du prochloraze, lorsque les souches de types la sont majoritaires (Curé et al, 1988; Migeon et Copin, 1988). Or, in vitro, les valeurs des $\mathrm{Cl}_{50}$ "mycélium» des souches de types Ils sont comprises entre 0,2 et $0,6 \mathrm{mg} / \mathrm{l}$ de flusilazole, ce qui leur confère des niveaux de résistance inférieurs ou égaux à 10 (comparativement aux souches de type la), (tableaux III et V).

Les comparaisons réalisées entre les souches résistantes au prochloraze induites en laboratoire (type Ic) et celles collectées dans la nature en 1990 (type llp) font apparaître des différences de comportement vis-à-vis des divers types d'inhibiteurs de la biosynthèse des stérols. Bien évidemment, la sensibilité des souches de type llp au fenpropimorphe et la résistance de celles de type Ic n'a rien de surprenant, car ces caractéristiques sont corrélées avec le type de croissance respectivement lente (type II) et rapide (type I) (Leroux et Gredt, 1988). Par contre, avec le prochloraze, le niveau d'expression de la résistance est différent entre les phénotypes lc et Ilp. En effet, chez les mutants de type Ic, la résistance est faiblement exprimée au niveau des hyphes mycéliens, alors qu'elle l'est fortement au moment de l'élongation des tubes germinatifs. Par contre, chez les souches de type Ilp, une résistance moyenne s'exprime à ces 2 stades. Par ailleurs, pour le clotrimazole et la terbinafine, la dissociation entre phénotypes lc et llp apparaît plus nettement, puisque la résistance vis-à-vis de ces 2 fongicides est exprimée chez le type lc, mais pas chez le type llp. Par contre, ces 2 types de souches résistantes au prochloraze ont comme point commun une stabilité de leurs caractères au cours du temps (observations sur une période de 6 mois avec des repiquages toutes les 3 semaines) en absence de tout fongicide (Leroux, résultats non publiés).

La comparaison des souches de type Ils et llp vis-à-vis des divers inhibiteurs de la C-14 déméthylation des stérols fait apparaître 3 types de comportement :

- résistance croisée positive pour le prochloraze, l'imazalile, le flusilazol, le flutriafol, le propiconazole et le tébuconazole,

- résistance croisée négative pour le triflumizole, le fénarimol, l'hexaconazole et le triadiménol, - absence de résistance croisée pour le clotrimazole, le diclobutrazole et le diniconazole. 
Un tel phénomène a été observée chez des mutants de Fusarium solani (Akallal, 1989; Kalamarakis et al, 1989) et chez des souches naturelles d'Helminthosporium teres (Kendall, 1986). Des études sont actuellement en cours pour déterminer si ces résultats sont corrélés à des modifcations qualitatives ou quantitatives de la cible : la C-14 déméthylase.

Enfin, la caractérisation en laboratoire des souches de $P$ herpotrichoides résistantes au prochloraze et pouvant se développer dans la nature, doit conduire à l'élaboration d'un système de diagnostic de cette résistance. Dans l'état actuel de nos connaissances celui-ci nécessite l'isolement des souches de $P$ herpotrichoides et par la suite, 2 méthodes sont applicables. La première que nous avons développée précédemment (Leroux et Gredt, 1988) fait appel à une observation de l'élongation des tubes germinatifs émis par les spores de $P$ herpotrichoides. Elle pourrait être simplifiée en utilisant une seule concentration de prochloraze (comprise entre 0,03 et $0,04 \mathrm{mg} / \mathrm{l}$ soit de l'ordre de $0,1 \mu \mathrm{mol} . .^{-1}$ ) au lieu des $2(0,01$ et $0,05 \mathrm{mg} / \mathrm{l})$ préconisées antérieurement. $\dot{A}$ cette concentration $(0,1 \mu \mathrm{mol}$. $\left.1^{-1}\right)$, parmi les souches naturelles, seules celles de type Ilp produisent des filaments germinatifs relativement longs (fig 2). La seconde méthode est basée sur l'effet du prochloraze au niveau de la croissance mycélienne de $P$ herpotrichoides cultivé sur milieu gélosé. Le criblage des souches pourrait se faire en présence de 0,5 et 2 $\mathrm{mg} / \mathrm{l}$ de prochloraze, ce qui correspond à des concentrations déjà utilisées par certains auteurs (King et al, 1986; Birchmore et al, 1987). Dans nos essais, à $0,5 \mathrm{mg} / \mathrm{l}$ la croissance mycélienne des phénotypes la, lb et lls est nulle ou réduite (au minimum $75 \%$ d'inhibition), tandis qu'à $2 \mathrm{mg} / \mathrm{l}$ aucun d'entre eux ne se développe (pour une incubation de 3 semaines). Quant aux souches de type $/ 1 \mathrm{p}$, à $0,5 \mathrm{mg} / \mathrm{l}$ leur croissance mycélienne n'est jamais réduite de plus de $50 \%$ et à $2 \mathrm{mg} / \mathrm{l}$ aucune d'entre elles n'est totalement inhibée (fig 1). Ces comportements s'observent lorsque $P$ herpotrichoides est cultivé sur milieu gélosé minéral à base de glucose et d'extrait de levure (voir : Matériels et méthodes) ou sur PDA (Difco) (Leroux, résultats non publiés). À notre connaissance, en Europe, toutes les souches rencontrées jusqu'à maintenant ont, sur milieu PDA, des $\mathrm{Cl}_{50}$ "mycelium» inférieures à $0,4 \mathrm{mg} / \mathrm{l}$ (Birchmore et al, 1990; N Cavelier, communication personnelle). Par contre, en Nouvelle-Zélande, King et al (1986) ont isolé, dès 1985, de nombreuses souches se développant en présence de $2 \mathrm{mg} / \mathrm{l}$ de prochloraze. Des analyses réalisées l'année suivante par Birchmore et al (1987) or qu'aucune souche ne se développait sur milieu PDA contenant $0,5 \mathrm{mg} / \mathrm{l}$ de prochloraze. Ces observations contradictoires suggèrent, soit qu'il y ait eu régression des souches résistantes au prochloraze, soit que la méthode de diagnostic utilisée par King et al (1986) était contestable.

\section{REMERCIEMENTS}

Nous tenons à remercier $D$ Paulmier du service régional de la protection des végétaux à Amiens qui a collecté les échantillons de blé atteints de piétin-verse à Ressons l'Abbaye et à Jaméricout (sites 1 et 2). Le suivi technique de l'essai implanté à Villiers-le-Bâcle (site 3) est réalisé par L Vignier de l'ITCF. Cette étude a bénéficié du soutien financier du ministère de la Recherche et de la Technologie (contrat $n^{\circ} 86 / 04$ ).

\section{RÉFÉRENCES}

Akallal R (1989) Résistance de Fusarium solani f sp pisi à des fongicides inhibant la $\mathrm{C}-14$ déméthylation des stérols. Thèse, Université de Paris-Sud (Orsay), $136 \mathrm{p}$

Andrivon D, Limpert E, Felsenstein FG (1987) Sensibilité au triadiménol et au fenpropimorphe de populations françaises d'Erysiphe graminis DC f sp hordei Marchal. Agronomie 7, 443-446

Birchmore RJ, Buckley ES, Browning S, Russell PE (1987) Sensitivity to prochloraz and carbendazim of New Zealand isolates of Pseudocercosporella spp. Aust Plant Pathol 16, 66-67

Birchmore RJ, Russell PE, Buschhaus H (1990) Long-term monitoring of Pseudocercosporella herpotrichoides populations for sensitivity to prochloraz and carbendazim. Brigh Crop Protec Conf Pests and Dis, 1153-1158

Butters J, Clark J, Hollomon DW (1984) Resistance to inhibitors of sterol biosynthesis in barley powdery mildew. Meded Fac Landbouww Rijksuniv Gent 49, 143-151

Cavelier N, Rousseau M, Le Page D (1987) Variabilité de Pseudocercosporella herpotrichoides, agent du piétin-verse des céréales : comportement in vivo de 2 types d'isolats et d'une population en mélange. Z Pflanzenkr Pflanzenschutz 94, 590-599

Curé B, Eschenbrenner P, Delalondre C (1988) Utilisation de prochloraze et du flusilazole pour lutter contre différentes souches de piétin-verse, sur blé d'hiver. $2^{\circ}$ conf int maladies plant, Ann ANPP, Bordeaux (France), 247-256

Fitt BDL, Goulds A, Hollins TW, Jones DR (1990) Strategies for the control of eyespot (Pseudocercosporella herpotrichoides) in UK winter wheat and winter barley. Ann Appl Biol 117, 473-486

Heanay SP (1988) Population dynamics of DMI fungicide sensitivity changes in barley powdery mildew. Fungicide resistance in North America (ed Delp CJ), APS Press, Saint-Paul (USA), 89-92 
Kalamarakis AE, Demopoulos VP, Ziogas BN, Georgopoulos SG (1989) A highly mutable major gene in Nectria haematoccoca var cucurbitae. Neth $J$ Plant Patho/ 95, suppl 1, 109-120

Kendall SJ (1986) Cross-resistance of triadimenolresistant fungal isolates to other sterol C-14 demethylation inhibitor fungicides. Brigh Crop Protec Conf Pests and Dis 539-546

King JE, Griffin MJ (1985) Survey of benomyl resistance in Pseudocercosporella herpotrichoides on winter wheat and barley in England and Wales in 1983. Plant Pathol 34, 272-283

King AC, Cole ALJ, Sanderson FR (1986) Prochloraz insensitivity in isolates of the cereal eyespot fungus Pseudocercosporella herpotrichoides, in New Zealand. Aust Plant Pathol 15, 22-23

Koller W (1988) Sterol demethylation inhibitors: mechanism of action and resistance. Fungicide resistance in North America (ed Delp CJ) APS Press, Saint Paul (USA), 79-88

Koller W, Scheinpflug $H$ (1987) Fungal resistance to sterol biosynthesis inhibitors: a new challenge. Plant Dis 71, 1066-1074

Lange de la Camp M (1966) Die Wirkungsweise von Cercosporella herpotrichoides. Fron, dem Erreger der Halmbruchkrankheit des Getreides. I. Feststellung der Krankheit, Beschaffenheit und Infektionsweise des Erregers. Phytopathol Z 55, 34-66

Leroux $P$ (1987) La résistance des champignons aux fongicides. Phytoma 385, 6-14

Leroux P (1989) Phénomènes de résistance des champignons phytopathogènes aux fongicides. $C R$ Acad Agric Fr 75, 37-44
Leroux P, Benveniste P (1985) Mode d'action des fongicides inhibiteurs des stérols. Fungicides for Crop Protection BCPC monograph $n^{\circ} 31$ (éd Smith IM), 67-68

Leroux P, Cavelier N (1983) Phénomènes de résistance du piétin-verse des céréales aux benzimidazoles et aux triophanates. Phytoma 351, 40-47

Leroux P, Gredt M (1988) Caractérisation des souches de Pseudocercosporella herpotrichoides, agent du piétin-verse des céréales, résistantes à des substances antimitotiques et à des inhibiteurs de la biosynthèse des stérols. Agronomie 8, 719729

Leroux P, Gredt M, Boéda P (1988) Resistance to inhibitors of sterol biosynthesis in field isolates or laboratory strains of the eyespot pathogen Pseudocercosporella herpotrichoides. Pestic Sci 23, 119-129

Marchegay P, Martin D, Guyot L (1988) Effets non intentionnels des traitements fongicides dans une succession colza-blé : objectifs et protocole. $2^{e}$ conf int maladies plant. Ann ANPP Bordeaux (France), 809-816

Migeon JL, Copin P (1988) Piétin-verse : caractérisation de situation en liaison avec le type de souches. $2^{\bullet}$ conf int maladies plant. Ann ANPP, Bordeaux (France), 293-300

Nirenberg HI (1981) Differenzierung der Erreger der Halmbruch-krankheit I. Morphologie. Z Pflanzenkr Pflanzenschutz 88, 242-248

Scott PR, Hollins TW, Muir P (1975) Pathogenicity of Cercosporella herpotrichoides to wheat, barley, oats and rye. Trans Br Mycol Soc 65, 529-538 